\title{
Estimación de parámetros forestales mediante datos de Sentinel 2A en Pueblo Nuevo, Durango
}

\section{Estimation of forest parameters using Sentinel 2A data in Pueblo Nuevo, state of Durango}

\author{
Pablito Marcelo López Serrano ${ }^{1}$, Daniel José Vega Nieva², Hugo Ramírez Aldaba²,

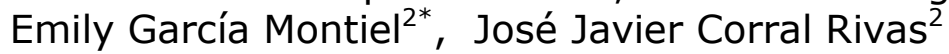

\begin{abstract}
The temperate forests demand periodic monitoring in order to reach a sustainable management. The remote sensing makes it possible to indirectly generate estimates under the assumption of a statistical correlation between satellite data and forest parameters. The aim of this work was to estimate the basimetric area $(G)$, the forest volume $(V t a)$ and the aboveground biomass $(W)$, using spectral data from the Sentinel 2A satellite in the San Bernardino de Milpillas Chico Community, Pueblo Nuevo, state of Durango. A correlation analysis was performed between mensuration information from 22 permanent plots for forest and soil research (SPIFyS) and high-resolution spectral information from the Sentinel 2A sensor. Subsequently, a multiple regression model was developed for each forest stand parameter. The highest correlation coefficient $(r)$ was observed in the NDVI with values of $0.77,0.68$ and 0.76 for the forest parameters of $V t a, G$ and $W$, respectively. The developed models explained $59 \%$ of the total variance observed for $V t a\left(R C M E=\mathrm{m}^{3}\right.$ ha $\left.{ }^{-1}\right), 58 \%$ for $W(R C M E=39.29$ $\left.\mathrm{Mg} \mathrm{ha}^{-1}\right)$ and $51 \%$ for $G\left(R C M E=4.40 \mathrm{~m}^{2} \mathrm{ha}^{-1}\right)$. The NDVI was the main predictive variable in three models. The Sentinel $2 \mathrm{~A}$ data with a resolution of $10 \mathrm{~m}$ in combination with mensuration information from SPIFyS showed a good capacity for mapping forest stand parameters in temperate forests.
\end{abstract}

Key words: Basimetric area, aboveground biomass, permanent plots, remote sensing, Sentinel, forest volume.

\section{Resumen}

Los bosques templados requieren de un monitoreo periódico con el fin de lograr un manejo sustentable. Los sensores remotos permiten hacer estimaciones de manera indirecta bajo el supuesto de que existe una correlación estadística entre datos satelitales y parámetros forestales. El objetivo del presente trabajo fue estimar el área basal $(G)$, el volumen forestal (Vta) y la biomasa forestal aérea (W) mediante datos espectrales del satélite Sentinel 2A en la Comunidad de San Bernardino de Milpillas Chico, Pueblo Nuevo, Durango. Se realizó un análisis de correlación entre información dasométrica procedente de 22 Sitios Permanentes de Investigación Forestal y de Suelos (SPIFyS) e información espectral de alta resolución del sensor Sentinel 2A. Posteriormente, se generó un modelo de regresión múltiple para cada parámetro forestal. El coeficiente de correlación (r) más alto se observó en el NDVI con valores de $0.77,0.68$ y 0.76 para los parámetros forestales de Vta, $G$ y $W$, respectivamente. Los modelos desarrollados explicaron $59 \%$ de la varianza total observada en el $V$ ta $\left(R C M E=57.60 \mathrm{~m}^{3} \mathrm{ha}^{-1}\right), 58 \%$ en $W\left(R C M E=39.29 \mathrm{Mg} \mathrm{ha}^{-1}\right)$, y $51 \%$ en $\mathrm{G}\left(R C M E=4.40 \mathrm{~m}^{2} \mathrm{ha}^{-1}\right)$. El NDVI fue la principal variable predictiva en los tres modelos. Los datos de Sentinel 2A con resolución de $10 \mathrm{~m}$ en combinación con información dasométrica derivada de SPIFyS mostraron una buena capacidad para el mapeo de parámetros forestales en bosques templados.

Palabras clave: Área basal, biomasa aérea, parcelas permanentes, sensores remotos, Sentinel, volumen forestal.

Fecha de recepción/Reception date: 18 de agosto de 2021

Fecha de aceptación/Acceptance date: 1 de enero de 2021

\footnotetext{
${ }^{1}$ Universidad Juárez del Estado de Durango. Instituto de Silvicultura e Industria de la Madera. México.

${ }^{2}$ Universidad Juárez del Estado de Durango. Facultad de Ciencias Forestales. México.
}

*Autor para correspondencia; correo-e: e_garcia@ujed.mx 


\section{Introducción}

Los bosques templados del estado de Durango, México son la principal fuente de producción de madera a nivel nacional (SRNyMA, 2016). De acuerdo con Segura y Trincado (2003), mantener en aprovechamiento y de manera sostenida a los bosques requiere de información actualizada y confiable de sus recursos naturales. Para lograr lo anterior, es necesario realizar un monitoreo periódico de estos ecosistemas (Tomppo et al., 2010). En ese sentido, el estudio de variables dasométricas para la investigación forestal permite dar seguimiento a la dinámica y estructura del ecosistema forestal (Gadow et al., 2012; Hernández-Ramos et al., 2020). Una forma de hacelo es por medio de los sitios permanentes que representan una base importante para obtener datos del efecto de la silvicultura en el crecimiento, producción y evolución de las masas forestales en lapsos cortos (Gadow et al., 1999). Sin embargo, dicha actividad generalmente conlleva a un tiempo largo de espera y altos costos para el establecimiento del arbolado y la colecta de información (Emborg, 1998; Toledo et al., 2011).

La aplicación de tecnologías geoespaciales es cada vez más relevante para estimar y monitorear parámetros forestales en periodos cortos (Foody et al., 2003; Hall et al., 2006; Fuchs et al., 2009; Verbesselt et al., 2010; Sobrino et al., 2019). De acuerdo con Herold et al. (2011) existe un particular interés en el manejo forestal del uso de sensores remotos para la estimación de atributos forestales, ya que favorecen la obtención de datos consistente, actualizada y espacialmente explícita en áreas de difícil acceso y con amplia cobertura. Al respecto, la estimación de parámetros forestales a partir de la combinación del uso de sensores remotos y de sitios de campo georreferenciados (sitios permanentes) se han convertido en técnicas útiles y confiables para estimar variables como el volumen forestal, el área basal y la biomasa forestal aérea (Hernández-Ramos et al., 2020; López-Serrano et al., 2020). 
Con base en esas tecnologías, dicha actividad se realiza de manera indirecta con el uso de técnicas estadísticas robustas bajo el supuesto de una alta correlación entre datos satelitales y datos del inventario tradicional (Aguirre-Salado et al., 2011; Song, 2013; Wulder et al., 2014; Acosta et al., 2017; López-Serrano et al., 2020).

Por otro lado, la disponibilidad y el mejoramiento de las capacidades de los diferentes tipos de sensores ofrecen la oportunidad de desarrollar técnicas de análisis que maximicen las estimaciones de los parámetros forestales, con información veraz que proviene de sitios permanentes de investigación forestal y de suelo, ya que fortalecen el insumo mediante sensores remotos (Gibbons y Chakraborti, 2003; Barajas, 2007; Karjalainen et al., 2012; Miranda-Aragón et al., 2013; Asner y Mascaro, 2014).

En función de lo anterior, el objetivo del presente trabajo consistió en estimar el área basal $(G)$, el volumen forestal ( $V t a)$ y la biomasa forestal aérea $(W)$ mediante datos espectrales del satélite Sentinel 2A en la Comunidad Indígena San Bernardino de Milpillas Chico, Pueblo Nuevo, Durango.

\section{Materiales y Métodos}

\section{Área de estudio}

El área de estudio se ubica en la Comunidad Indígena de San Bernardino de Milpillas Chico, ubicada en el municipio Pueblo Nuevo, Durango, México (Figura 1). La Comunidad cuenta con una superficie de 156 618.33 ha. Presenta climas de tipo cálido subhúmedo $(\mathrm{CW})$, cálido semifrío $[\mathrm{C}(\mathrm{E}) \mathrm{x}]$; la temperatura media del mes más frío es de $3^{\circ} \mathrm{C}$ a $18^{\circ} \mathrm{C}$ y del mes más caliente de $6.5^{\circ} \mathrm{C}$ a $22^{\circ} \mathrm{C}$, con una precipitación media anual de $1300 \mathrm{~mm}$. Los tipos de suelo presentes en el ejido son Regosol, Fluvisol y Cambisol, someros y pedregosos. Su intervalo altitudinal comprende de los 2500 a los 2600 m (Inegi, 2017b). El tipo de vegetación 
corresponde a bosque de pino, en la que las especies arbóreas dominantes son Pinus durangensis Martínez, Pinus teocote Schltdl. \& Cham., Pinus leiphylla Schltld. \& Cham. y Pinus cooperi C.E. Blanco var. cooperi (Inegi, 2017a).
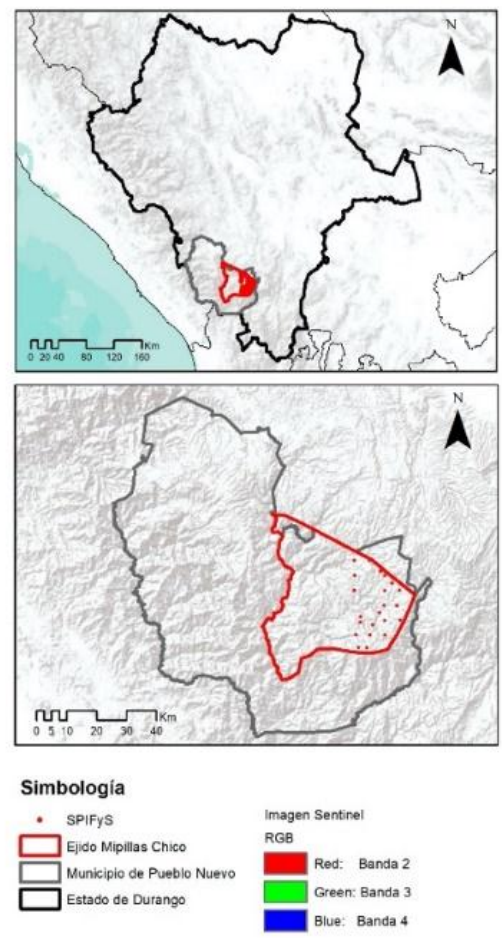

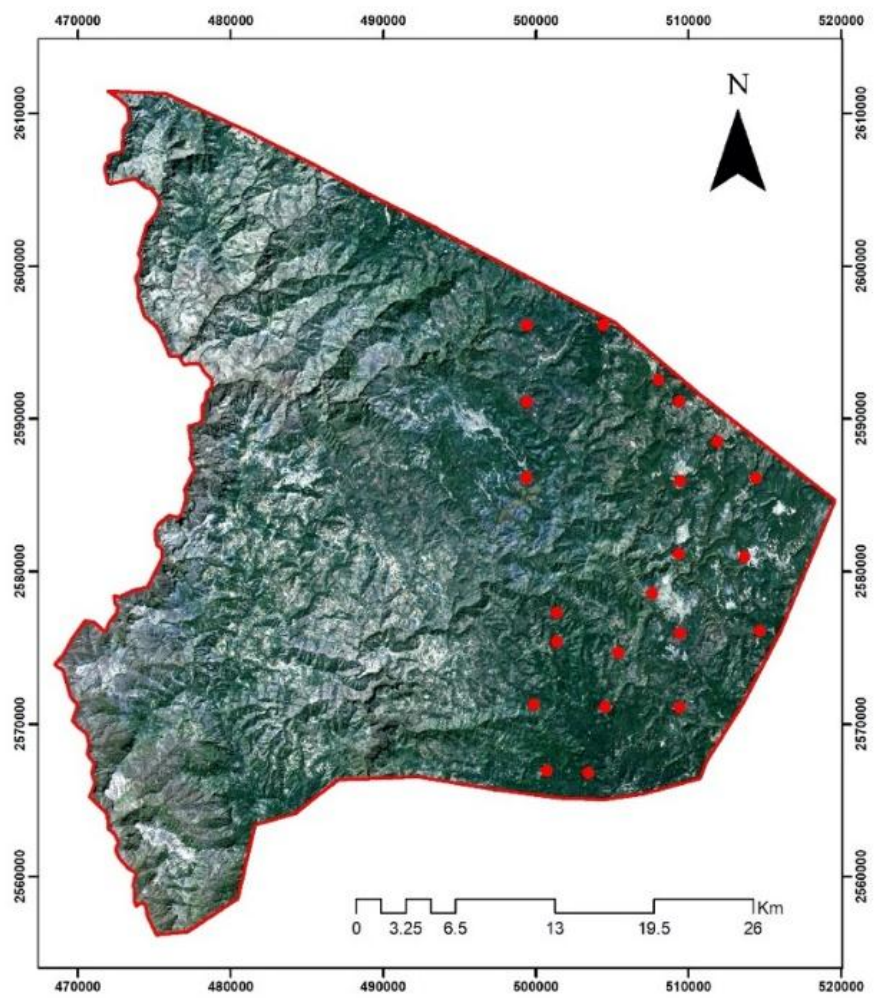

Figura 1. Localización del área de estudio. 


\section{Datos de campo}

Los datos dasométricos se obtuvieron de 22 Sitios Permanentes de Investigación Forestal y Suelos (SPIFyS) establecidos durante el invierno del año 2009 mediante la metodología desarrollada por Corral-Rivas et al. (2009); y posteriormente remedidos en intervalos de 5 años (2014 y 2019). Los SPIFyS miden $50 \times 50$ m y se ubicaron mediante un muestreo sistemático, con una distancia promedio de 3 a 5 $\mathrm{km}$ entre ellos. Para el cálculo del área basal $(G)$, se utilizaron las técnicas de modelización forestal descritas por Diéguez-Aranda et al. (2005). El volumen (Vta) y la biomasa $(W)$ se calcularon con las ecuaciones específicas por especie desarrolladas por Simental-Cano et al. (2017) y Vargas-Larreta et al. (2017), respectivamente.

\section{Adquisición y procesamiento de las imágenes de satélite}

Se adquirieron y procesaron tres escenas de satélite del sensor Sentinel-2A (Cuadro 1) del servidor del Servicio Geológico de Estados Unidos (USGShttps://glovis.usgs.gov). Dichas imágenes tienen un procesamiento de nivel 1 (Level 1C), de las cuales solo se utilizaron las bandas del sector visible e infrarrojo, con la misma resolución espacial (Cuadro 2). A fin de eliminar los efectos de la atmósfera, se procesaron las imágenes para obtener valores de reflectancia de la superficie (SR-Level 2A), mediante la herramienta Sen2Cor (Casella et al., 2018) en Software de la Plataforma de Aplicación Sentinel 2A (SNAP) (Louis et al., 2016). Posteriormente, se calculó el Índice de Vegetación de Diferencia Normalizada (NDVI), con el propósito de contribuir con la estimación de los parámetros forestales. 
$N D V I=(N I R-R) /(N I R+R)$

Donde:

$N I R=$ Banda espectral en la región del infrarrojo cercano

$R=$ Banda en la región rojo

Cuadro 1. Características de las imágenes del sensor Sentinel $2 \mathrm{~A}$, utilizadas en este estudio.

Identificador Fecha de adquisición $\begin{aligned} & \text { Cobertura de nubes } \\ & (\%)\end{aligned}$ Agencia

\begin{tabular}{llll}
\hline T13QDF & $22 / 11 / 2019$ & 0.02 & ESA \\
T13QEF & $22 / 11 / 2019$ & 0 & ESA \\
T13QDG & $22 / 11 / 2019$ & 4.2 & ESA \\
\hline
\end{tabular}

$E S A=$ Agencia Espacial Europea.

Cuadro 2. Características de las bandas del sensor Sentine/ $2 \mathrm{~A}$, utilizadas en este estudio.

\begin{tabular}{llll}
\hline Banda & $\begin{array}{l}\text { Longitud de } \\
\text { onda }(\boldsymbol{\mu m})\end{array}$ & Resolución $(\mathbf{m})$ & Abreviatura \\
\hline Azul & $0.45-0.52$ & 10 & B1 \\
Verde & $0.54-0.57$ & 10 & B2 \\
Rojo & $0.65-0.68$ & 10 & B3 \\
Infrarrojo cercano & $0.78-0.90$ & 10 & IRC \\
\hline
\end{tabular}




\section{Análisis estadístico}

Se realizó un análisis de correlación para determinar la relación entre variables espectrales y parámetros forestales. Posteriormente, se ajustaron modelos de regresión lineal múltiple para identificar a las variables que predicen mejor los parámetros forestales mediante el procedimiento stepwise (selección por pasos), bajo la estrategia mixta; es decir, se usó una combinación de la selección forward y backward, con la librería MASS (Ripley, 2020), en el programa R Core Team (2020).

El modelo utilizado fue de la forma:

$$
y=\beta_{0}+\beta_{1} X_{1}+\beta_{2} X_{2}+\ldots+\beta_{n} X_{n}+\varepsilon_{j}
$$

Donde:

$y=$ Parámetro forestal por estimar

$X_{n}=$ Bandas espectrales e índice de vegetación

$\beta_{n}=$ Coeficientes de regresión

$\varepsilon_{i}=$ Error aleatorio

$G, V t a$ y $W=$ Variables dependientes

Bandas $B 1, B 2, B 3$ e $I C R$ y $N D V I<0=$ Variables independientes

Para evaluar la capacidad de ajuste del modelo se calcularon los coeficientes de bondad del ajuste, coeficiente de determinación ajustado $\left(R_{A d j}^{2}\right)$ y la raíz del cuadrado medio del error ( $R C M E)$. 
$R^{2}{ }_{A d j}=1-\left[\frac{n-1 \sum_{i=1}^{n}\left(y_{i}-\mathrm{y}_{i}\right)^{2}}{n-\mathrm{p}-1 \sum_{i=1}^{n}\left(y_{i}-\bar{y}\right)^{2}}\right]$

$R C M E=\sqrt{\left[\frac{\sum_{i=1}^{n}\left(y_{i}-\hat{y}_{i}\right)^{2}}{n-p}\right\rceil}$

Donde:

$y_{i}=$ Valor observado de la variable dependiente estudiada

$\hat{y}_{i}=$ Valores predicho de la variable dependiente estudiada

$\bar{y}_{i}=$ Media de la variable dependiente estudiada

$n$ = Número de observaciones totales

$p=$ Número de parámetros del modelo

Una vez evaluado el mejor modelo, se le utilizó para generar los mapas de cada parámetro forestal; para ello, se consideró solo el área de bosque templado para el área de estudio, con base en el uso de suelo y vegetación (Inegi, 2017a); este proceso se llevó a cabo mediante la librería raster (Hijmans, 2020). Enseguida, se generó la distribución espacial del error del modelo (residuos) de cada parámetro con una interpolación de Distancia Inversa Ponderada (IDW), por medio de la librería gstat (Pebesma, 2004). Dichos procesos se hicieron en el programa $R$ ( $R$ Core Team, 2020). En la Figura 2 se muestra el diagrama de flujo de trabajo del presente estudio. 


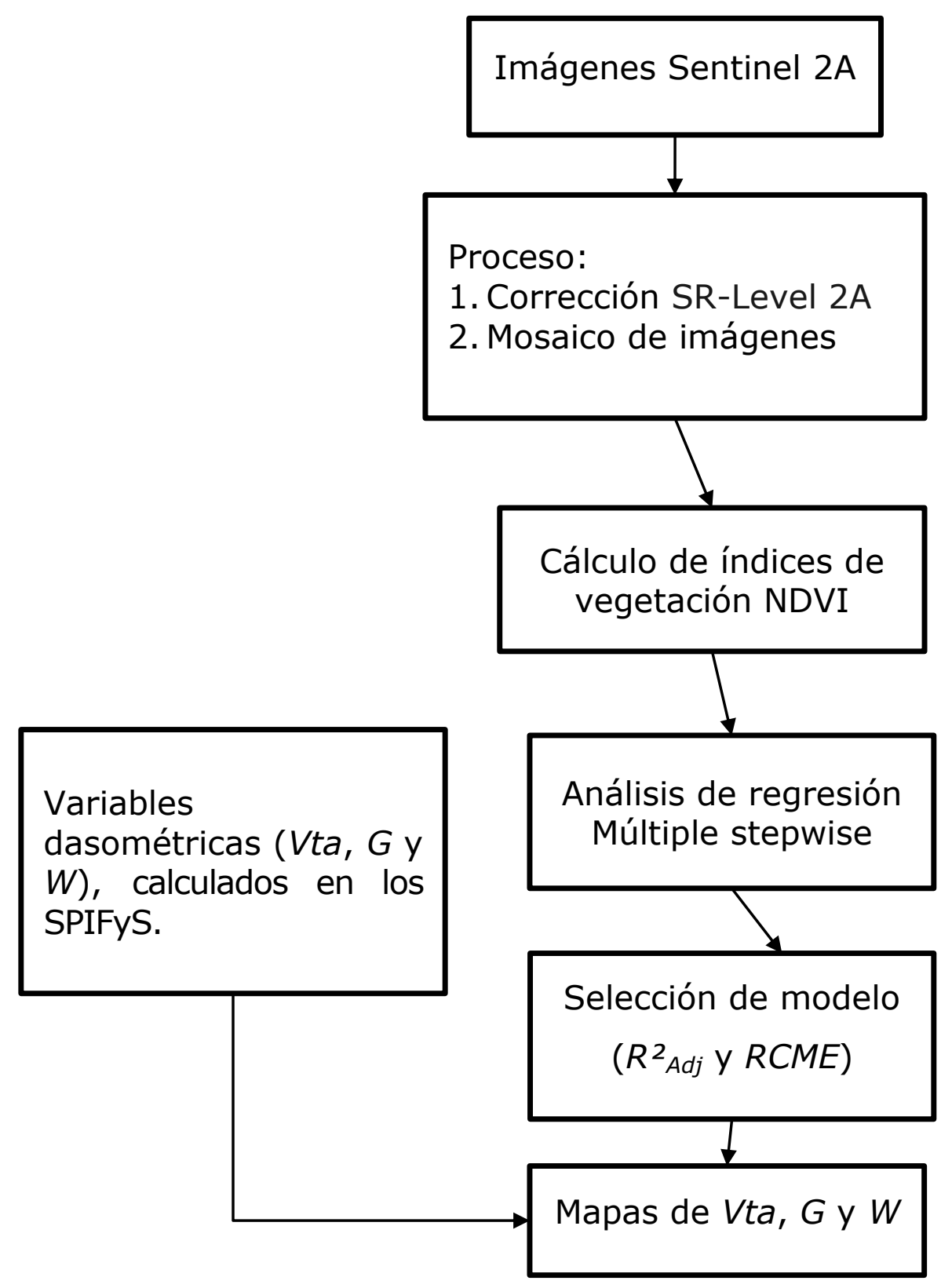

Figura 2. Flujo de trabajo utilizado en este estudio. 


\section{Resultados y Discusión}

Las principales estadísticas descriptivas para las variables dasométricas por hectárea en los sitios de estudio se resumen en el Cuadro 3. Los resultados muestran que en la Comunidad de San Bernardino de Milpillas Chico el área basal por hectárea $(G)$ se distribuye en un intervalo de 11.23 a $34.98 \mathrm{~m}^{2} \mathrm{ha}^{-1}$, con un valor medio de $19.82 \mathrm{~m}^{2} \mathrm{ha}^{-1}$. Vta y $W$ tuvieron valores de 97.38 a $418.62 \mathrm{~m}^{3} \mathrm{ha}^{-1}$, y de 54.271 a $289.418 \mathrm{Mg} \mathrm{ha}^{-1}$, con promedios de $198.037 \mathrm{~m}^{3} \mathrm{ha}^{-1}$ y $121.683 \mathrm{Mg}$ $\mathrm{ha}^{-1}$, respectivamente. Estos resultados fueron similares a los obtenidos por Graciano-Ávila (2019) y López-Serrano (2020) para este tipo de bosques en la región del mismo municipio, Pueblo Nuevo, Durango, México.

Cuadro 3. Estadísticas descriptivas de los parámetros forestales estimados en los 22 SPIFys evaluados.

\begin{tabular}{lllll}
\hline Variable & Mínimo & Máximo & Media & StD \\
\hline$G$ & 11.23 & 34.98 & 19.82 & 6.63 \\
$V t a$ & 97.38 & 418.62 & 198.04 & 97.22 \\
$W$ & 54.271 & 289.42 & 121.68 & 65.52 \\
$G=$ Área basal $\left(\mathrm{m}^{2} \mathrm{ha}^{-1}\right) ;$ Vta = Volumen forestal $\left(\mathrm{m}^{3} \mathrm{ha}^{-1}\right) ; W=$ Biomasa forestal \\
aérea $\left(\mathrm{Mg} \mathrm{ha}^{-1}\right) ;$ StD $=$ Desviación estándar.
\end{tabular}

La correlación entre $G, V t a$ y $W$ de cada SPIFyS con las diferentes bandas espectrales y NDVI se presentan en la Figura 3. El coeficiente de correlación de Pearson ( $r$ ) varió de -0.26 a 0.77 . El análisis registró una asociación negativa en las reflectancias de las bandas espectrales B1, B2 y B3 con los parámetros forestales; mientras que el IRC y el NDVI tuvieron tendencias positivas. El valor de $r$ más alto se registró en el NDVI de $0.68,0.77$ y 0.76 para $G$, Vta y $W$, respectivamente; 
comportamiento que resulta similar al publicado por diversos autores bajo el mismo objetivo de estimación de variables dasométricas, con distintos tipos de sensores en diferentes masas forestales (López-Serrano et al., 2016; Acosta et al., 2017; Dos et al., 2018; Hernández-Ramos et al., 2020).

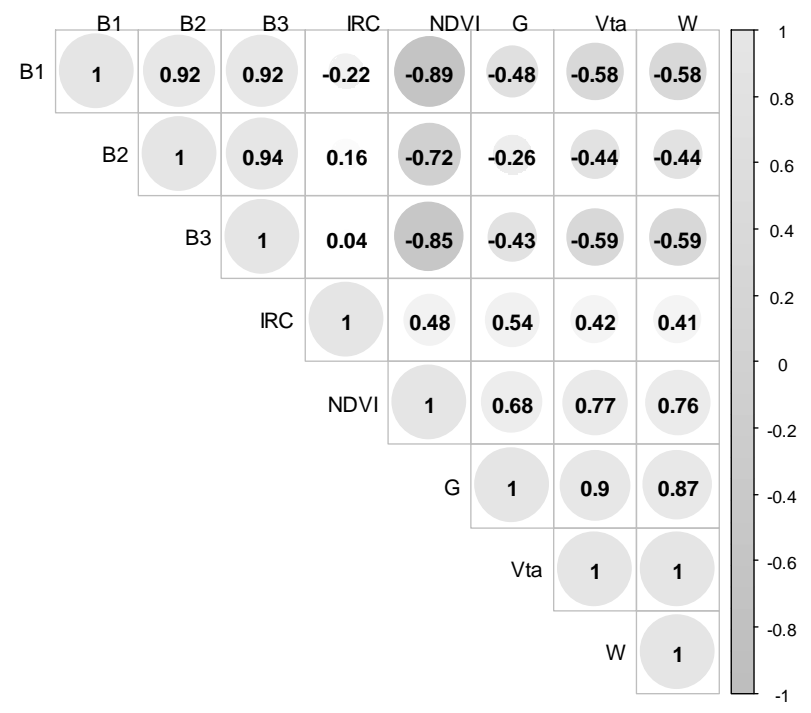

Figura 3. Coeficientes de correlación de Pearson entre variables espectrales y parámetros forestales.

La correlación alta observada en el NDVI $(r=>0.60)$ con cada una de las variables dependientes, se debe a que el NDVI tiene la capacidad de explicar la variación de la vegetación fotosintéticamente activa dada la combinación de reflectancia en el espectro verde e infrarrojo del sector electromagnético (Chuvieco, 2002; Lu et al., 2004). Esto lo convierte en el índice de vegetación más utilizado como predictor en la estimación de parámetros biofísicos forestales, dada la creciente accesibilidad a la información espectral con resoluciones espaciales cada vez más finas (Assmann et al., 2020; Myers-Smith et al., 2020). 
Los valores de los coeficientes de determinación ajustados $\left(R^{2}{ }_{A d j}\right)$ y errores de los mejores modelos (RCME) para estimar los parámetros forestales en el área de estudio se resumen en el Cuadro 4. En la Figura 4, se ilustra la distribución de los residuales de los modelos. El estadístico $R^{2}{ }_{\text {Adj }}$ de los modelos de regresión estimados varió de 0.51 a 0.59 . En la estimación de $V$ ta, el valor de $R^{2}{ }_{\text {Adj }}$ fue ligeramente mayor, ya que el modelo logró explicar $59 \%$ de la varianza total observada en ese atributo $\left(R C M E=57.60 \mathrm{~m}^{3} \mathrm{ha}^{-1}\right)$. Sin embargo, su valor es ligeramente inferior al obtenido por Chrysafis et al. (2017), quienes estimaron el volumen forestal en ecosistemas forestales del mediterráneo, y se basaron en imágenes Sentinel-2, aunque en su caso calcularon un error superior al estimado en este trabajo $\left(R^{2}=0.63 ; R C M E=63.11 \mathrm{~m}^{3} \mathrm{ha}^{-1}\right)$ y Landsat 8 OLI $\left(R^{2}=0.62 ; R C M E\right.$ $=64.40 \mathrm{~m}^{3} \mathrm{ha}^{-1}$ ) y $R C M E$ mayor. 
Cuadro 4. Modelos de regresión utilizados en este estudio con sus respectivos estadísticos de ajuste.

\begin{tabular}{lcccc}
\hline Modelo & $\boldsymbol{\beta}$ & Valor & $\boldsymbol{R}^{2}{ }_{\text {Adj }}$ & $\boldsymbol{R C M E}$ \\
\hline & $\beta_{0}$ & -33.096 & & \\
& $\beta_{1}$ & 0.03543 & 0.51 & 4.4 \\
& $\beta_{2}$ & 65.5993 & & \\
& $\beta_{0}$ & -1478.4 & & \\
$V t a=\beta_{0} \beta_{1} B 3+\beta_{2} I C R+\beta_{3} N D V I$ & $\beta_{1}$ & 1.4387 & 0.59 & 57.6 \\
& $\beta_{2}$ & -0.3673 & & \\
& $\beta_{3}$ & 2801.5 & & \\
& $\beta_{0}$ & -1021.2 & & \\
$W=\beta_{0}+\beta_{1} B 3+\beta_{2} I C R+\beta_{3} N D V I$ & $\beta_{1}$ & 0.9901 & & \\
& $\beta_{2}$ & -0.2546 & & \\
& $\beta_{3}$ & 1915.4 & & \\
\hline
\end{tabular}

$G=$ Área basal $\left(\mathrm{m}^{2} \mathrm{ha}^{-1}\right) ; v t a=$ Volumen forestal $\left(\mathrm{m}^{3} \mathrm{ha}^{-1}\right) ; W=$ Biomasa forestal $\left(\mathrm{Mg} \mathrm{ha}^{-1}\right) ; \beta=$ Parámetros del modelo; $R^{2}{ }_{A d j}=$ Coeficiente de determinación ajustado; $R C M E=$ Raíz del cuadrado medio del error. 

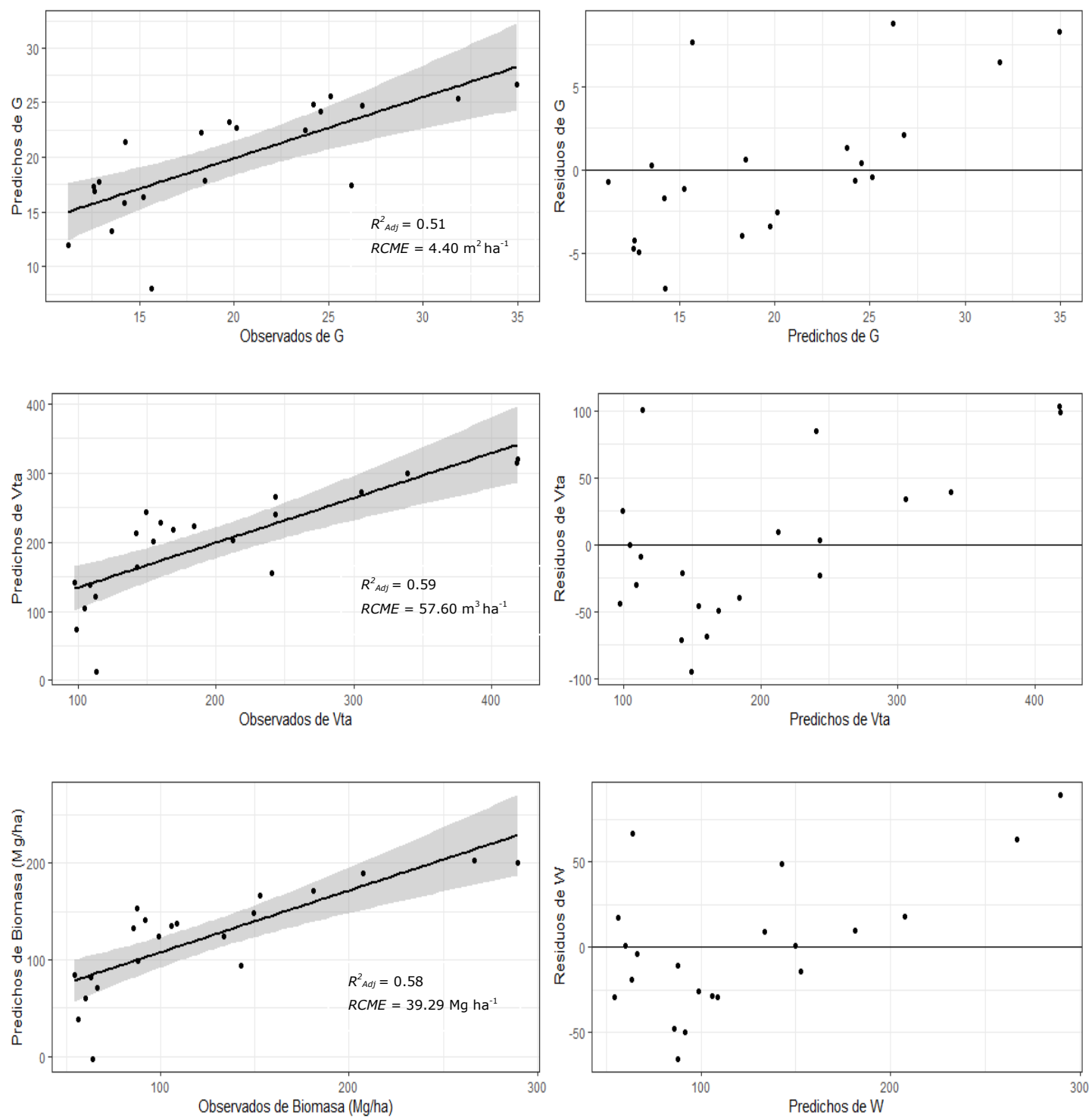

Figura 4. Valores predichos frente a valores observados de los modelos seleccionados para la estimación de los parámetros forestales estudiados.

Hu et al. (2020) estimaron el volumen forestal mediante un análisis de regresión lineal múltiple $\left(R^{2}=0.49 ; R C M E=70.22 \mathrm{~m}^{3} \mathrm{ha}^{-1}\right)$, basado en las variables derivadas de Sentinel-2, en los bosques de la provincia de Hunan, China. 
En el caso particular de México, estos resultados fueron superiores a los de Hernández-Ramos et al. (2020), quienes calcularon el volumen $\left(R^{2}{ }_{A d j}=0.32 ; R C M E\right.$ $\left.=68.39 \mathrm{~m}^{3} \mathrm{ha}^{-1}\right)$, el área basal $\left(R^{2}{ }_{A d j}=0.28 ; R C M E=7.64 \mathrm{~m}^{2} \mathrm{ha}^{-1}\right)$ y la biomasa $\left(R^{2}{ }_{A d j}=0.32 ; R C M E=35.65 \mathrm{Mg} \mathrm{ha}^{-1}\right)$, bajo una técnica estadística de regresión lineal múltiple, en diferentes ecosistemas de selvas mediante la combinación de información espectral de media resolución (Landsat) e información derivada del Inventario Nacional Forestal y de Suelos (INFyS) de Quintana Roo.

Por otra parte, Torres-Vivar et al. (2017) calcularon el Vta $\left(R^{2}{ }_{A d j}=0.66 ; R C M E=\right.$ $\left.62.3 \mathrm{~m}^{3} \mathrm{ha}^{-1}\right), G\left(R_{A d j}^{2}=0.66 ; R C M E=5.82 \mathrm{~m}^{2} \mathrm{ha}^{-1}\right)$ y $W\left(\mathrm{R}^{2}{ }_{\mathrm{Adj}}=0.66 ; R C M E=32\right.$ $\mathrm{Mg} \mathrm{ha}^{-1}$ ) en bosques de coníferas en el estado de Hidalgo, a partir de un análisis de regresión múltiple y datos del sensor de alta resolución SPOT 6. Bajo el mismo esquema, en ecosistemas de selva baja caducifolia y con datos de media resolución (Landsat) en el Estado de México, Acosta et al. (2017) determinaron valores del $R C M E$ para Vta de $13.18 \mathrm{~m}^{3} \mathrm{ha}^{-1}\left(R_{A d j}^{2}=0.66\right)$, mientras que para $G$ un valor de $3.30 \mathrm{~m}^{2} \mathrm{ha}^{-1}\left(R_{A d j}^{2}=0.52\right)$ y para $W 5.91 \mathrm{Mg} \mathrm{ha}^{-1}\left(R_{A d j}^{2}=0.60\right)$; cifras que fueron superiores a las del presente trabajo.

Dicha variación en los resultados del $R C M E$ y $R^{2}$ Adj en la estimación y monitoreo de la vegetación con sensores remotos podrían atribuirse a la resolución espacial de las imágenes, a las condiciones ambientales prevalecientes durante su toma, e incluso al tipo de vegetación en cada estudio de caso (López-Serrano et al., 2016; TorresRojas et al.,2016; Hawryło et al., 2018; Pham et al., 2019; Hernández-Ramos et al., 2020; López-Serrano et al., 2020).

Finalmente, una vez seleccionado el mejor modelo se generaron los mapas para cada parámetro forestal estudiado y se graficó la distribución espacial del error de dicho modelo (Figura 5). Para el caso del $G$ la distribución espacial en el área de estudio varió de 0 a $40 \mathrm{~m}^{2} \mathrm{ha}^{-1}$, para el Vta de 0 a $500 \mathrm{~m}^{3} \mathrm{ha}^{-1}$ y $W$ de 0 a $300 \mathrm{Mg}$ 
$\mathrm{ha}^{-1}$. Estos mapas representan un esquema de la distribución del recurso forestal que se pueden integrar al plan de manejo forestal para mejorar su gestión.
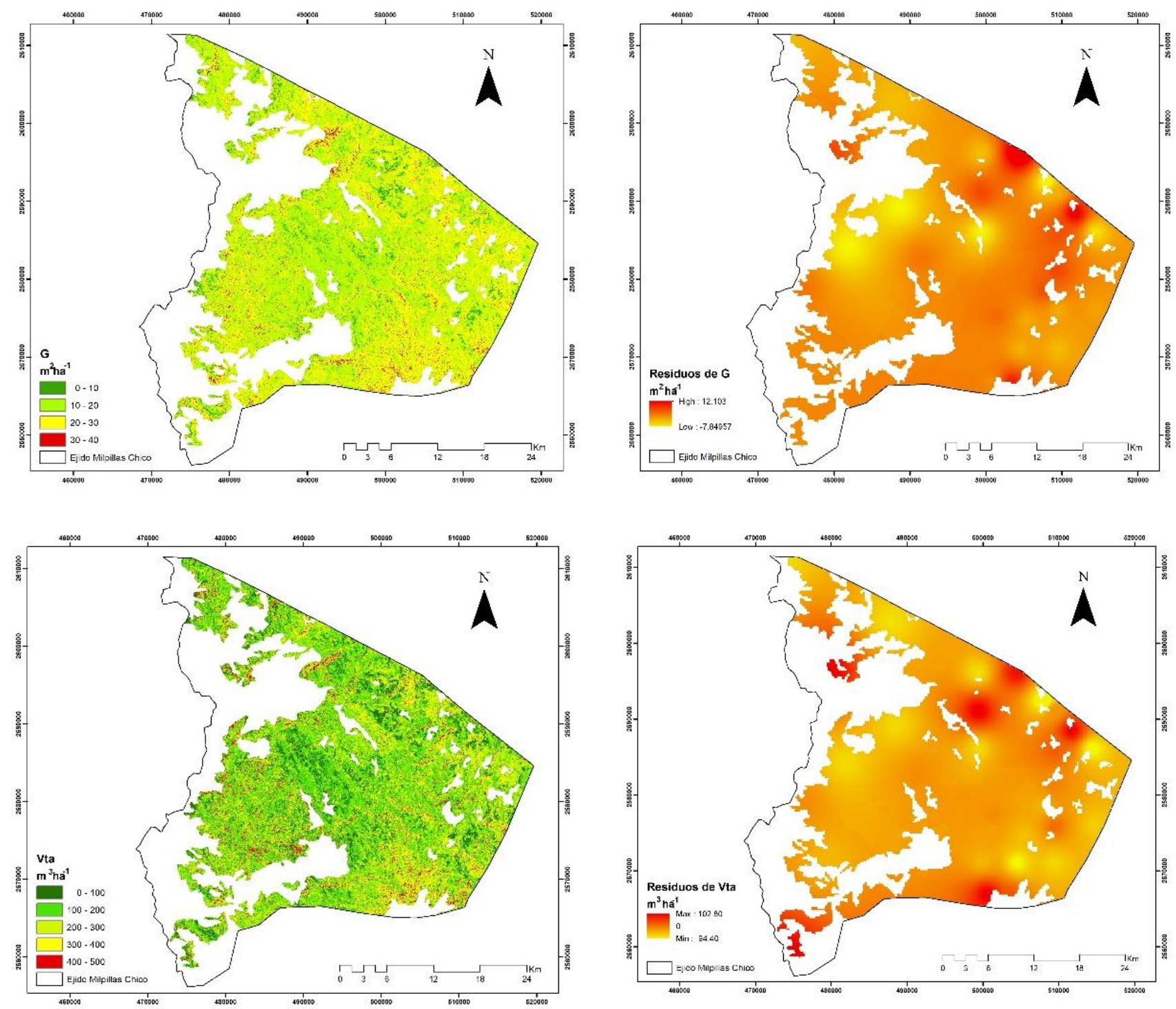

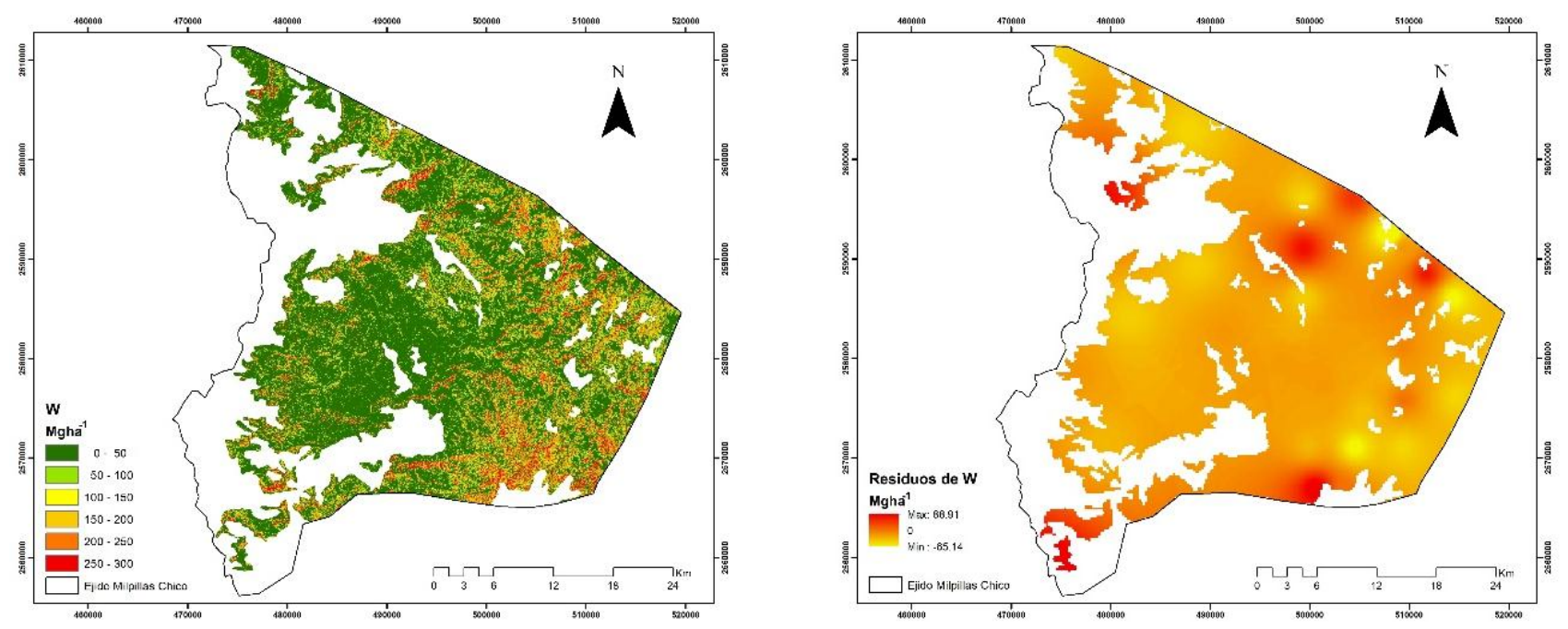

Figura 5. Estimación espacial y distribución espacial del error de $G, V t a$ y $W$ en los bosques de la Comunidad Indígena de San Bernardino de Milpillas Chico. 


\section{Conclusiones}

La generación de modelos de regresión permitió estimar de manera indirecta el $G$, el Vta y la $W$ mediante información espectral derivada del sensor Sentinel 2A e información dasométrica derivada de SPIFyS. El índice de vegetación NDVI es la variable espectral que presenta mayor correlación con los parámetros forestales estudiados (0.68-0.77). Las imágenes de alta resolución del sensor Sentinel 2A son una herramienta útil para el mapeo de parámetros forestales en bosques templados a nivel regional.

\section{Agradecimientos}

Al Programa para el Desarrollo Profesional Docente, para el tipo Superior (Prodep) a través del proyecto 123-UJED.

\section{Conflicto de intereses}

Los autores declaran no tener conflicto de intereses.

\section{Contribución por autor}

Pablito Marcelo López Serrano: análisis estadístico, escritura del manuscrito; Daniel José Vega Nieva: revisión y corrección del manuscrito; Hugo Ramírez Aldaba y Emily García Montiel: revisión y coordinación del proceso de edición; José Javier Corral Rivas: muestreo en campo, metodología y revisión de manuscrito. 


\section{Referencias}

Acosta M., M. R., S. M. E. Pérez, Romero, H. A., González, y A. L. Martínez. 2017. Estimación de la densidad forestal mediante imágenes Landsat ETM+ en la región sur del Estado de México. Revista Mexicana de Ciencias Forestales 8(41): 30-55. Doi:10.29298/rmcf.v8i41.25.

Aguirre-Salado, C. A., J. R. Valdez-Lazalde, G. Ángeles-Pérez, H. M. de los SantosPosadas y A. I. Aguirre-Salado. 2011. Mapeo del índice de área foliar y cobertura arbórea mediante fotografía hemisférica y datos SPOT 5 HRG: regresión y k-nn. Agrociencia 45(1): 105-119.

http://www.scielo.org.mx/scielo.php?script=sci_arttext\&pid=S1405$\underline{31952011000100010}$ (2 de marzo de 2021).

Asner G. P. and J. Mascaro. 2014. Mapping tropical forest carbon: Calibrating plot estimates to a simple LiDAR metric. Remote Sensing of Environment 140:614-624. Doi: $10.1016 /$ j.rse.2013.09.023.

Assmann, J. J., I. H. Myers-Smith, J. T. Kerby, A. M., Cunliffe and G. Daskalova. 2020. Drone data reveal heterogeneity in tundra greenness and phenology not captured by satellites. Environmental Research Letters 15(12): 125002. Doi: 10.1088/1748-9326/abbf7d.

Barajas F., H. 2007. Comparación entre análisis discriminante no-métrico y regresión logística multinomial. Tesis de Maestría, Facultad de Ciencias, Universidad Nacional de Colombia. Medellín, Colombia. 67 p.

Casella, A., N. Barrionuevo, A. Pezzola y C. Winschel. 2018. Preprocesamiento de imágenes satelitales del sensor Sentinel $2 A$ y $2 B$ con el software SNAP 6. 0. Instituto de Clima y Agua. CIRN INTA Castelar. Buenos Aires, Argentina. pp. 1-31. 
Chrysafis, I., G. Mallinis, S. Siachalou and P. Patias. 2017. Assessing the relationships between growing stock volume and Sentinel-2 imagery in a Mediterranean forest ecosystem. Remote Sensing Letters 8: 508-517. Doi: 10.1080/2150704X.2017.1295479.

Chuvieco, E. 2002. Teledetección Ambiental. La observación de la Tierra desde el Espacio. Editorial Ariel. Barcelona, España. 616 p.

Corral-Rivas, J. J., B. Vargas L., C. Wehenkel, O. A. Aguirre C., J. G. Álvarez G. y A. Rojo A. 2009. Guía para el Establecimiento de Sitios de Investigación Forestal y de Suelos en Bosques del Estado de Durango. Editorial UJED. Durango, Dgo., México. $81 \mathrm{p}$.

Diéguez-Aranda, U., F. Castedo D. y J. Álvarez G. 2005. Funciones de crecimiento en área basimétrica para masas de Pinus sylvestris L. procedentes de repoblación en Galicia. Investigación Agraria. Sistemas y Recursos Forestales 14(2): 253-266. http://www.inia.es/gcontrec/pub/253-266-(143 04)-

Funciones 1162281545765.pdf (2 de marzo de 2021).

Dos R., A. A., M. C. Carvalho, J. M. De Mello, L. R. Gomide, A. C. Ferraz F. and F. W. A. Junior. 2018. Spatial prediction of basal area and volume in Eucalyptus stands using Landsat TM data: an assessment of prediction methods. New Zealand Journal of Forestry Science 48(1): 1-17. Doi: https://doi.org/10.1186/s40490-017-0108-0.

Emborg, J. 1998. Understorey light conditions and regeneration with respect to the structural dynamics of a near-natural temperate deciduous forest in Denmark. Forest Ecology and Management 106: 83-95. Doi: 10.1016/S0378-1127(97)00299$\underline{5}$.

Foody, G. M., D. S. Boyd and M. E. J. Cutler. 2003. Predictive relations of tropical forest biomass from Landsat TM data and their transferability between regions. Remote Sensing of Environment 85: 463-474. Doi:10.1016/S0034-4257(03)00039‥ 
Fuchs, H., P. Magdon, K. Kleinn and H. Flessa. 2009. Estimating aboveground carbon in a catchment of the Siberian forest tundra: Combining satellite imagery and field inventory. Remote Sensing of Environment 113(3): 518-531.

Doi: 10.1016/j.rse.2008.07.017.

Gadow, K. V., A. Rojo, G. Álvarez-González y R. Rodríguez-Soalleiro. 1999. Ensayos de crecimiento. Parcelas permanentes, temporales y de intervalo. Investigación Agraria. Sistemas y Recursos Forestales 1:299-310.

https://recyt.fecyt.es/index.php/IA/article/view/2776 (2 de marzo de 2021).

Gadow, K. V., C. Y. Zhang, C. Wehenkel, A. Pommerening, J. Corral R., M. Korol and X. H. Zhao. 2012. Forest structure and diversity. In: Pukkala, T. and K. von Gadow (eds.). Continuous cover forestry. Springer. Dordrecht, Netherlands. pp. 29-83. Doi: 10.1007/978-94-007-2202-6 2 .

Gibbons, J. D. and S. Chakraborti. 2003. Nonparametric Statistical Interference; Marcel Denker, Inc. New York, NY, USA. 645 p.

Graciano-Ávila, G., E. Alanís-Rodríguez, O. A. Aguirre-Calderón, M. González-Tagle, E. J. Treviño-Garza, A. Mora-Olivo y E. Buendía-Rodríguez. 2019. Estimación de volumen, biomasa y contenido de carbono en un bosque de clima templado-frío de Durango, México. Revista Fitotecnia Mexicana 42(2): 119-127. http://www.scielo.org.mx/pdf/rfm/v42n2/0187-7380-rfm-42-02-119.pdf (2 de marzo de 2021).

Hall, R. J., R. S. Skakun, E. J. Arsenault and B. S. Case. 2006. Modeling forest stand structure attributes using Landsat ETM+ data: Application to mapping of aboveground biomass and stand volume. Forest Ecology and Management 225: 378-390. Doi: 10.1016/j.foreco.2006.01.014.

Hawryło, P., B. Bednarz, P. Wężyk and M. Szostak. 2018. Estimating defoliation of Scots pine stands using machine learning methods and vegetation indices of 
Sentinel-2. European Journal of Remote Sensing 51(1): 194-204.

Doi: https://doi.org/10.1080/22797254.2017.1417745.

Hernández-Ramos, J., X. García-Cuevas, R. Peréz-Miranda, A. González-Hernández y L. Martínez-Ángel. 2020. Inventario y mapeo de variables forestales mediante sensores remotos en el estado de Quintana Roo, México. Madera y Bosques 26(1):e2611884. Doi:10.21829/myb.2020.2611884.

Herold, M., R. M. Román-Cuesta, D. Mollicone, Y. Hirata, P. Van Laake, G. P. Asner, C. Souza, M. Skutsch, V. Avitabile and K. Macdicken. 2011. Options for monitoring and estimating historical carbon emissions from forest degradation in the context of REDD+. Carbon Balance and Management 6: 1-13. Doi:10.1016/j.rse.2009.08.014. Hijmans, R. J. 2020. Raster: Geographic Data Analysis and Modeling. R package version 3.4-5. https://CRAN.R-project.org/package=raster (9 de abril de 2021). Hu, Y., X. Xu, F. Wu, Z. Sun, H. Xia, Q. Meng and X. Xiao. 2020. Estimating forest stock volume in Hunan Province, China, by integrating in situ plot data, Sentinel-2 images, and linear and machine learning regression models. Remote Sensing 12(1): 186. Doi: $10.3390 /$ rs12010186.

Instituto Nacional de Estadística y Geografía (Inegi). 2017a. Anuario estadístico y geográfico de Durango.

https://www.datatur.sectur.gob.mx/ITXEF Docs/DGO ANUARIO PDF.pdf (15 de julio de 2020).

Instituto Nacional de Estadística y Geografía (Inegi). 2017b. Conjunto de datos vectoriales de uso del suelo y vegetación Escala 1: 250 000. Serie VI (Conjunto nacional). URL:

http://www.conabio.gob.mx/informacion/metadata/gis/usv250s6gw.xml? httpcache =yes\& $\mathrm{xs}=/ \mathrm{db} / \mathrm{metadata} / \mathrm{xs} / \mathrm{fgdc} \mathrm{html} . \mathrm{xs} / \&$ indent=no (2 de marzo de 2021). 
Karjalainen, M., V. Kankare, M. Vastaranta, M. Holopainen and J. Hyyppa. 2012. Prediction of plot-level forest variables using TerraSAR-X stereo SAR data. Remote Sensing of Environment 117: 338-347. Doi:10.1016/j.rse.2011.10.008.

López-Serrano, P. M., C. A. López S., R. Solís-Moreno and J. J. Corral-Rivas. 2016. Geospatial estimation of above ground forest biomass in the Sierra Madre Occidental in the state of Durango, Mexico. Forests 7(3): 70.

\section{Doi: $10.3390 /$ f7030070.}

López-Serrano, P. M., C. A. López-Sánchez, J. G. Álvarez-González and J. GarcíaaGutiérrez. 2016. A comparison of machine learning techniques applied to landsat-5 TM spectral data for biomass estimation. Canadian Journal of Remote Sensing 42(6): 690-705. Doi: 10.1080/07038992.2016.1217485.

López-Serrano, P. M., J. L. Cárdenas D., J. J. Corral-Rivas, E. Jiménez, C. A. LópezSánchez and D. J. Vega-Nieva. 2020. Modeling of aboveground biomass with Landsat $8 \mathrm{OLI}$ and machine learning in temperate forests. Forests 11(1): 11. Doi: $10.3390 / f 11010011$.

Louis, J., V. Debaecker, B. Pflug, M. Main-Knorn, J. Bieniarz, J., U. Mueller-Wilm and F. Gascon. 2016. Sentinel-2 Sen2Cor: L2A processor for users. In: Proceedings Living Planet Symposium. 9-13 May 2016. Prague, Czech Republic. 8 p.

Lu, D., P. Mausel, E., Brondízio and E. Moran. 2004. Relationships between forest stand parameters and Landsat TM spectral responses in the Brazilian Amazon Basin. Forest Ecology and Management 198: 149-167. Doi: 10.1016/j.foreco.2004.03.048. Miranda-Aragón, L., E. J. Treviño-Garza, J. Jiménez-Pérez, O. A. Aguirre-Calderón, M. A. González-Tagle, M. Pompa-García y C. A. Aguirre-Salado. 2013. Tasa de deforestación en San Luis Potosí, México (1993-2007). Revista Chapingo Serie Ciencias Forestales y del Ambiente 19(2): 201-215.

Doi:10.5154/r.rchscfa.2011.06.044. 
Myers-Smith, I.H., J. T. Kerby, G. K. Phoenix, J. W. Bjerke, H. E. Epstein, J. J. Assmann, C. J., L Andreu-Hayles, S. Angers-Blondin, P. S. A. Beck, L. T. Berner, U. S. Bhatt, A. D. Bjorkman, D. Blok, A. Bryn, C. T. Christiansen, J. H. C. Cornelissen, A. M. Cunliffe, S. C. Elmendorf, B. C. Forbes, S. J. Goetz, R. D. Hollister, R. Jong, M. M. Loranty, M. Macias-Fauria, K. Maseyk, S. Normand, J. Olofsson, T. C. Parker, F. W. Parmentier, E. Post, G. Schaepman-Strub, F. Stordal, P. F. Sullivan, H. J. D. Thomas, H. Tømmervik, R. Treharne, C. E. Tweedie, D. A. Walker, M. Wilmking and S. Wipf. 2020. Complexity revealed in the greening of the Arctic. Nature Climate Change 10(2): 106-117. Doi:10.1038/s41558-019-0688-1.

Pebesma, E. J. 2004. Multivariable geostatistics in S: the gstat package. Computers and Geosciences 30: 683-691. Doi: 10.1016/j.cageo.2004.03.012.

Pham, T., N. Yokoya, D. Bui, K. Yoshino and D. Friess. 2019. Remote sensing approaches for monitoring mangrove species, structure, and biomass: Opportunities and challenges. Remote Sensing 11:230. Doi: 10.3390/rs11030230.

Ripley, B. 2020. MASS: Support Functions and Datasets for Venables and Ripley's Mass. https://CRAN.R-project.org/package=MASS (2 de marzo de 2021).

R Core Team. 2020. R: A language and environment for statistical computing. $R$ Foundation for Statistical Computing, Vienna, Austria. https://www.R-project.org/ (9 de abril de 2021).

Secretaría de Recursos Naturales y Medio Ambiente (SRNyMA). 2016. Programa Estratégico Forestal 2030. Gobierno del Estado de Durango. Durango, Dgo., México. $200 \mathrm{p}$.

Segura, M. R. y G. Trincado. 2003. Cartografía digital de la Reserva Nacional Valdivia a partir de imágenes satelitales Landsat TM. Bosque (Valdivia) 24(2):4352. Doi: https://dx.doi.org/10.4067/S0717-92002003000200005.

Simental-Cano, B., C. A. López-Sánchez, C. Wehenkel, B. Vargas-Larreta, J. G. Álvarez-González and J. J. Corral-Rivas. 2017. Species-specific and regional volume 
models for 12 forest species in Durango, Mexico. Revista Chapingo Serie Ciencias Forestales y del Ambiente 3(2): 155-171. Doi: 10.5154/r.rchscfa.2016.01.004. Sobrino J., A., R. Llorens, C. Fernández, J. M. Fernández A. and A. Vega J. 2019. Relationship between soil burn severity in forest fires measured in situ and through spectral indices of remote detection. Forests 10(5): 457. Doi: 10.3390/f10050457. Song, C. 2013. Optical remote sensing of forest leaf area index and biomass. Progress in Physical Geography 37: 98-113. Doi: 10.1177/0309133312471367. Toledo, M., L. Poorter, M. P. Claros, A. Alarcon, J. Balcázar, C. Leaño, J. C. Licona, O. Llanque, V. Vroomans, P. Zuidema and F. Bongers. 2011. Climate is a stronger driver of tree and forest growth rates than soil and disturbance. Journal of Ecology 99(1): 254-264. Doi: 10.1111/j.1365-2745.2010.01741.x.

Tomppo, E., Th. Gschwantner, M. Lawrence and E. McRoberts. 2010. National Forest Inventories - Pathways for Common Reporting. Springer book series Managing Forest Ecosystems. Viena, Austria. 612 p. Doi: 10.1007/978-90-481-3233-1. Torres-Rojas, G., M. E. Romero-Sánchez, E. Velasco-Bautista y A. GonzálezHernández. 2016. Estimación de parámetros forestales en bosques de coníferas con técnicas de percepción remota. Revista Mexicana de Ciencias Forestales 7(36): 724. Doi:10.29298/rmcf.v7i36.56.

Torres-Vivar, J. E., J. J. Valdez-Lazalde, G. Ángeles P., H. M. Santos-Posadas y C. A. Aguirre-Salado. 2017. Inventario y mapeo de un bosque bajo manejo de pino con datos del sensor SPOT 6. Revista Mexicana de Ciencias Forestales 8(39): 25-43. Doi:10.29298/rmcf.v8i39.41.

Vargas-Larreta, B., C. A. López-Sánchez, J. J. Corral-Rivas, J. O. López-Martínez, C. G. Aguirre-Calderón and J. G. Álvarez-González. 2017. Allometric equations for estimating biomass and carbon stocks in the temperate forests of North-Western Mexico. Forests 8(8): 269. Doi: 10.3390/f8080269. 
Verbesselt, J., R. Hyndman, G. Newnham and D. Culvenor. 2010. Detecting trend and seasonal changes in satellite image time series. Remote Sensing of Environment 114: 106-115. Doi: https:10.1016/j.rse.2009.08.014. Wulder, M. A., S. M. Ortlepp, J. C. White and S. Maxwell. 2014. Evaluation of Landsat-7 SLC-off image products for forest change detection. Canadian Journal of Remote Sensing 34(2): 93-99. Doi: 10.5589/m08-020. se distribuyen amparados bajo la licencia Creative Commons 4.0 Atribución-No Comercial (CC BY-NC 4.0 Internacional), que permite a terceros utilizar lo publicado siempre que mencionen la autoría del trabajo y a la primera publicación en esta revista. 